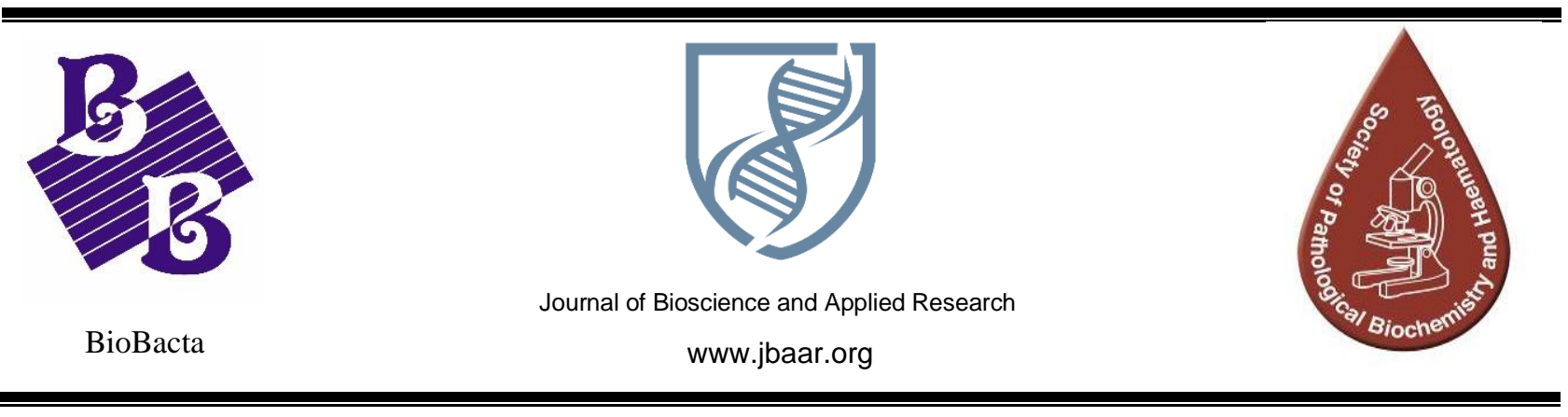

\title{
Tissue inhibitor metalloproteinase-1, Plasminogen activator inhibitor 1, and neutrophil/lymphocyte ratio as potential early biomarkers for diabetic nephropathy
}

\author{
Mohamed M. Omran ${ }^{1, *}$, Rasha A. Youssef ${ }_{2}^{2}$ Fathy M. Eltaweel ${ }^{2}$, \\ Ashraf A. Tabll ${ }^{3}$, Ahmed A. Eldeeb ${ }^{4}$ \\ ${ }^{1}$ Chemistry Department, Faculty of Science, Helwan University, Cairo, Egypt \\ ${ }^{2}$ Chemistry Department, Faculty of Science, Damietta University, Egypt \\ ${ }^{3}$ Microbial Biotechnology Department, National Research Centre, Giza, Egypt \\ ${ }^{4}$ Nephrology Unit, Faculty of Medicine, Mansoura University, Mansoura, Egypt \\ Short Title: Predicting diabetic nephropathy
}

DOI: $10.21608 /$ jbaar.2019.147125

\author{
*Correspondence: \\ Mohamed Mostafa Omran, PhD \\ Chemistry Department, Faculty of Science, \\ Helwan University, Ain Helwan, 11795 \\ Cairo, Egypt, \\ E-mail: drmmomran@yahoo.com
}

Scopus ID): https://www.scopus.com/authid/detail.uri?authorId=8622008300

ORCID id: http://orcid.org/0000-0001-8557-3104 


\section{Abstract}

Background: Albuminuria is used to screen early stages of diabetic nephropathy (DN) but it is limited by the fact that structural damage may precede albumin excretion. This necessitates identifying better biomarkers that diagnose or predict diabetic nephropathy. The study aimed to evaluate tissue inhibitor metalloproteinase-1 (TIMP-1), plasminogen activator inhibitor 1 (PAI-1), and neutrophil/lymphocyte ratio (NLR) as potential biomarkers for early detection of diabetic nephropathy and its progression in patients with type 2 diabetes.

Materials and Methods: A total of 88 subjects were included in this cross-sectional hospital-based study, healthy individuals $(\mathrm{N}=10)$ and diabetic patients $(\mathrm{n}=78)$. Diabetic patients were classified according to an albumin creatinine ratio $(\mathrm{ACR})$ into normoalbuminuria $(\mathrm{A} 1=30)$, microalbuminuria $(\mathrm{A} 2=14)$, and macroalbuminuria $(\mathrm{A} 3$ =34). TIMP-1, PAI-1 levels, NLR were measured in all subjects. Multivariate discriminant analysis (MDA) was used to develop a novel index. The diagnostic value of TIMP-1, PAI-1, and NLR was assessed by the area under the receiver operating characteristic (ROC). Results: The mean \pm SD of NLR, PAI-1 (ng/ml) and TIMP-1 (ng/ml) in healthy were $(1.9 \pm 0.30 ; 6.8 \pm 2.4$ and $76.8 \pm 15.7)$ and in A1 were $(3.0 \pm 2.5 ; 7.7 \pm 1.8$ and $91.4 \pm 19.8)$ and in A2 were ( $2.2 \pm 1.5 ; 7.6 \pm 1.9$ and $104.5 \pm 20.9)$ and in $A 3$ were $(5.2 \pm 3.9 ; 8.7 \pm 1.2$ and $120.6 \pm 18.2)$. The differences between the mean of NLR, PA1-1 and, TIMP in A2 and that of A3 were significant ( $<<0.007, p=0.03$ and, $p<$ 0.011; respectively). TIMP-1 was the most efficient marker with an AUC of 0.72 for discriminant diabetics with A1 from A2; 0.88 for $\mathrm{A} 1$ vs $\mathrm{A} 3$ and 0.82 for $\mathrm{A} 2 \mathrm{vs} \mathrm{A} 3$. A novel index was developed for differentiated between stages of DN based on three blood markers (TIMP-1, PAI-1, and NLR) named TPN. The mean \pm SD of TPN index in healthy was $(1.1 \pm 0.4)$ and in A1 was $(1.2 \pm 0.3)$ and in A2 was $(1.3 \pm 0.2)$ and in A3 were $(1.8 \pm 0.3)$ with a highly significant difference between $\mathrm{A} 2$ and $\mathrm{A} 3$. The AUC of the TPN index was $0.61,0.88$, and 0.88 for discriminant diabetics with A1 from A2, A1 vs A3, and A2 vs A3. Conclusions: A novel index named TPN based on three blood markers (TIMP-1, PAI-1, and NLR) may be potentially useful for early detection and to discriminate macro-albuminuria from micro-albuminuria stages.

Keywords: Type 2 diabetes; diabetic nephropathy; diagnosis; Blood markers; early stages; late stages

\section{Introduction:}

Worldwide, diabetes is the most common metabolic disorder; about $35 \%$ of patients with diabetes develop diabetic nephropathy (Dias et al., 2018). The therapeutic outcome of diabetic nephropathy is unable to prevent disease progression (Kim and Park, 2017). This is due to the low sensitivity of available markers such as microalbuminuria and creatinine clearance (Nah et al., 2017). Several diagnostic biomarkers predicting the early stages of $\mathrm{DN}$ in patients with diabetes have been identified and are becoming increasingly important in clinical diagnostics (AlRubeaan et al., 2017; Vijay et al., 2018). Important biomarkers associated with early DN in diabetic patients are neutrophil gelatinase-associated lipocalin (NGAL), N-acetyl-beta-glucosaminidase (NAG), Cystatin C, alpha 1-microglobulin, immunoglobulin G/or M, type IV collagen, nephrin, angiotensinogen, and lever-type fatty acid-binding protein (Fiseha, 2015; Zeng et al., 2017). NLR was a marker in chronic kidney diseases (Kuo et al., 2018). Our work focuses on three biomarkers (TIMP-1, PAI-1, and NLR). TIMP-1 influences various biological processes including cell growth, apoptosis, promoting cell proliferation, and this by acting as a signaling molecule in its own right (Musial and Zwolińska, 2014; Polina et al., 2018). It was demonstrated that imbalance 
between activity of both matrix metalloproteinases (MMP) and tissue inhibitors of metalloproteinases (TIMPs) is involved in DN. Expression of MMPs is decreased and expression of TIMPs is increased by high glucose level (McLennan et al., 2004). Plasminogen activator inhibitor-1 (PAI-1) has been associated with coronary heart diseases and with matrix accumulation in chronic inflammatory kidney diseases (Małgorzewicz et al., 2013; Baker et al., 2018). The NLR is a blood marker used as an indicator of chronic kidney inflammation and its value increase with the severity of CKD (Tatar et al., 2016). This work aimed to evaluate whether TIMP-1, PAI-1, and NLR can be used as single and combined diagnostic biomarkers to screen for diabetic nephropathy and its progression in type 2 diabetic subjects.

\section{Materials \& methods}

\section{Patients:}

A cross-sectional study was performed on 88 subjects (33 males, 55 females; mean age 56.3 \pm 11.4 years). Patients were selected from attendants of the endocrinology and nephrology units and outpatient clinics of Mansoura university hospital, Mansoura, Egypt. These subjects were classified into three groups: ten non-diabetic healthy subjects (5 males, 5

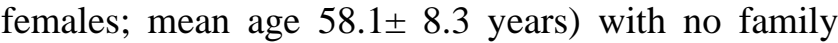
history of diabetes, and diabetic patients $(n=78)$. Diagnosis of diabetes was done based on the American Diabetes Association criteria (American Diabetes Association, 2018). Diabetic patients were classified according to an albumin creatinine ratio (ACR) into normoalbuminuria $(\mathrm{A} 1=30), \quad$ microalbuminuria $\quad(\mathrm{A} 2=14), \quad$ and macroalbuminuria ( $\mathrm{A} 3=34$ ) (Guy et al., 2019). The assessment was carried out by collecting the following clinical and demographic variables for each patient: full personal history, age (years), sex, history of diabetes (duration and complications) and arterial pressure, clinical examination including abdominal examination and abdominal ultrasound, and CT scan. Excluded subjects were having type-1 diabetes mellitus, and other causes of proteinuria (urinary tract infection, severe hypertension, and hematuria).

\section{Samples:}

Blood was obtained from each subject and collected in the morning after fasting overnight and another sample was collected two hours after breakfast. A portion of the blood was treated immediately with KEDTA as an anti-coagulant for a complete blood picture (CBC) and Glycosylated hemoglobin (HBA1c). Another portion of blood samples was allowed to clot at room temperature before centrifugation for 15 minutes at $(1000 \times \mathrm{g})$ to collect the serum. Serum samples were stored at $-80 \mathrm{C}$ until analysis. The firstmorning urine specimen was collected from all participants. Urine samples were examined for albumin creatinine ratio (ACR) (Guy et al., 2019). Diabetic patients were divided into three groups by urinary albumin-to-creatinine ratio (ACR): normoalbuminuria group (ACR $<30 \mathrm{mg} / \mathrm{g}$ ), microalbuminuria (ACR 30$300 \mathrm{mg} / \mathrm{g}$ ) and macroalbuminuria (ACR $>300 \mathrm{mg} / \mathrm{g}$ ). The value of eGFR was calculated according to MDRD GFR Equation (Levey et al., 2014).

\section{Biochemical analysis:}

All participants were subjected to routine laboratory investigations, including complete blood picture (using automated hematology analyzer (KX-21 Sysmes, System Corporation, Japan)), fasting blood sugar, kidney function tests, and lipid profile using an automated biochemistry analyzer (BT1500; Biotecnica instruments S.P.A, Italy). HBA1c was measured by using an automated analyzer (Labona Check Alc, SYNAPSE, Belgium). NLR was calculated as a simple ratio between the absolute neutrophil and the absolute lymphocyte counts both obtained from the same automated blood sample at the admission of the study. Serum PAI-1 and TIMP-1 levels were assayed by using an ELISA kit (Elabscience, Wuhan, Hubei, China), and 
an absorbance reader (Tecan Austria GmbH, Sunrise Basic TECAN) at $450 \mathrm{~nm}$ was used to measure the developed color absorbance.

\section{Data analysis:}

Qualitative and quantitative data were analyzed using the Statistical Package of Social Sciences (SPSS) system (version 19.0). Comparisons of quantitative variables were done with ANOVA test, $t$-test while the Mann-Whitney $U$ test was used in cases with nonparametric variables. The correlations between variables were analyzed with the Spearman correlation coefficient. Multivariate discriminant analysis (MDA) was used to develop the novel index. The diagnostic value of TIMP-1, PAI-1, and NLR was assessed by the area under the receiver operating characteristic (ROC). The best cutoff points were selected and diagnostic powers were determined.

\section{Results:}

Laboratory data of studied groups were presented in table 1. Using t-test, there were significant differences ( $\mathrm{p}$ values ranged from 0.001- 0.0001) between $\mathrm{A} 2$ and $\mathrm{A} 3$ in kidney function tests, HBA1C, GFR, and ACR. The mean \pm SD of NLR, PAI-1 (ng/ml) and TIMP-1 $(\mathrm{ng} / \mathrm{ml})$ in healthy were $(1.9 \pm 0.30 ; 6.8$ \pm 2.4 and $76.8 \pm 15.7)$ and in A1 were $3.0 \pm 2.5 ; 7.7 \pm 1.8$ and $91.4 \pm 19.8)$ and in $\mathrm{A} 2$ were $2.2 \pm 1.5 ; 7.6 \pm 1.9$ and $104 \pm 20.9)$ and in $A 3$ were $(5.2 \pm 3.9 ; 8.7 \pm 1.2$ and $120 \pm 18.2$ ). There were significant differences between $\mathrm{A} 2$ and A3 in NLR (p 0.007), PAI1 (0.03) and TIMP1 (0.031); respectively.

\section{Correlation among TIMP-1, PAI-1, and other tests:}

Linear significant correlations were observed between TIMP-1 levels and kidney function tests that included: creatinine ( $\mathrm{r}=0.418 ; \mathrm{p}<0.0001)$, uric acid ( $\mathrm{r}$ $=0.469 ; \mathrm{p}<0.0001)$, BUN ( $\mathrm{r}=0.503 ; \mathrm{p}<0.0001)$, and
eGFR ( $r=-0.488 ; p<0.0001)$. Linear significant correlation were observed between PAI-1 and eGFR ( $\mathrm{r}$ $=-0.348 ; \mathrm{p}<0.001)$.

\subsection{Diagnostic power of NLR, TIMP-1, and PAI-1:}

Using the ROC curve, we assessed the diagnostic powers of NLR, TIMP-1, and PAI-1 to discriminate among different study groups was presented in table 2 . TIMP-1 was the most efficient marker $(\mathrm{AUC}=0.72$, $71.4 \%$ sensitivity and 66.7 specificity) for discriminant diabetics with $\mathrm{A} 1$ from $\mathrm{A} 2$; for $\mathrm{A} 1 \mathrm{vs} \mathrm{A} 3$ ( $\mathrm{AUC}=0.88$, with $81.8 \%$ sensitivity and $8.3 \%$ specificity ) and for A2 vs A3 (AUC $=0.82$ with $81.8 \%$ sensitivity and $78.6 \%$ specificity); table 2 .

\section{Development of a novel index for discriminant between DN stages}

We developed a new index for differentiated between DN stages based on three blood markers (TIMP-1, PAI-1, and NLR) named TPN. It can be calculated as $((0.006 \times$ TIMP-1 $)+(0.104 \times$ PAI- 1$)+$ $(0.004 \times$ NLR $)-0.078))$. The levels of the TPN index were increased with the severity of $\mathrm{DN}$. The mean $\pm \mathrm{SD}$ of TPN index in healthy was $(1.1 \pm 0.4)$ and in A1 was $(1.2 \pm 0.3)$ and in $\mathrm{A} 2$ was $1.3 \pm 0.2)$ and in A3 were $(1.8 \pm 0.3)$ with a highly significant difference between $A 2$ and A3 ( $<<0.0001)$. The diagnostic power of the TPN was tested for three clinically relevant thresholds of DN (A1, A2, and A3). The AUC of the TPN index was 0.61 with $64.3 \%$ sensitivity and $63.3 \%$ specificity for discriminated patients with A1 from A2. For discriminated patients with A1 vs A3, AUC was 0.88 with $81.8 \%$ sensitivity and $80 \%$ specificity. Also, for discriminated patients with A2 vs A3, AUC was 0.88 with $81.8 \%$ sensitivity and $85.7 \%$ specificity at best cutoff $=1.48$ (i.e. less than 1.48 indicated microalbuminuria and greater than 1.48 indicated macroalbuminuria) 
Table 1. Laboratory data of studied groups

\begin{tabular}{|c|c|c|c|c|c|c|}
\hline \multicolumn{2}{|c|}{ Variables } & Healthy $(n=10)$ & A1 $(n=30)$ & A2 $(n=14)$ & A3 (n=34) & P-value * \\
\hline \multirow[t]{2}{*}{ Gender } & male & $5(50.0 \%)$ & $14(46.7 \%)$ & $3(21.4 \%)$ & $11(32.4 \%)$ & \multirow[b]{2}{*}{0.449} \\
\hline & Female & $5(50.0 \%)$ & $16(53.3 \%)$ & $11(78.6 \%)$ & $23(67.6 \%)$ & \\
\hline \multirow{2}{*}{\multicolumn{2}{|c|}{$\begin{array}{l}\text { Age (years) } \\
\text { Neutrophils }\left(10^{9} / 1\right)\end{array}$}} & $38.1 \pm 8.3$ & $55.8 \pm 10.4$ & $57.9 \pm 9.0$ & $60.7 \pm 8.8$ & 0.328 \\
\hline & & $59.7 \pm 4.4$ & $60.9 \pm 15.4$ & $57.3 \pm 14.8$ & $71.9 \pm 11.6$ & 0.001 \\
\hline \multicolumn{2}{|c|}{ Lymphocytes $\left(10^{9} / 1\right)$} & $31.9 \pm 4.8$ & $28.4 \pm 12.9$ & $33.3 \pm 12.2$ & $19.4 \pm 9.4$ & $<0.0001$ \\
\hline \multicolumn{2}{|c|}{ Creatinine (mg/dl) } & $0.7 \pm 0.1$ & $0.7 \pm 0.1$ & $1.1 \pm 0.2$ & $3.6 \pm 1.9$ & $<0.0001$ \\
\hline \multicolumn{2}{|c|}{ Uric acid (mg/dl) } & $3.4 \pm 0.3$ & $4.8 \pm .9$ & $4.7 \pm 2.5$ & $10.2 \pm 3.6$ & $<0.0001$ \\
\hline \multicolumn{2}{|c|}{ Cholesterol (mg/dl) } & $122.5 \pm 15.4$ & $154.3 \pm 58.2$ & $162.8 \pm 49.2$ & $164.4 \pm 56.3$ & 0.939 \\
\hline \multicolumn{2}{|c|}{ Triglycerides (mg/dl) } & $73.4 \pm 8.5$ & $144.0 \pm 66.9$ & $170.3 \pm 74.9$ & $159.0 \pm 91.1$ & 0.746 \\
\hline \multicolumn{2}{|c|}{ HDL-Ch $(\mathrm{mg} / \mathrm{dl})$} & $69.2 \pm 5.5$ & $55.1 \pm 11.3$ & $36.2 \pm 12.9$ & $41.2 \pm 13.0$ & 0.336 \\
\hline \multicolumn{2}{|c|}{ LDL-Ch (mg/dl) } & $38.6 \pm 13.1$ & $74.2 \pm 47.5$ & $97.7 \pm 39.6$ & $100.2 \pm 48.5$ & 0.890 \\
\hline \multicolumn{2}{|c|}{ F.B.G $(\mathrm{mg} / \mathrm{dl})$} & $89.1 \pm 4.9$ & $157.0 \pm 47.7$ & $182.6 \pm 123.4$ & $175.1 \pm 99.7$ & 0.849 \\
\hline \multicolumn{2}{|c|}{$\operatorname{HBA} 1 \mathrm{C}(\%)$} & $4.9 \pm 0.2$ & $8.9 \pm 2.7$ & $9.1 \pm 2.9$ & $6.5 \pm 1.17$ & 0.001 \\
\hline \multicolumn{2}{|c|}{$\mathrm{ACR}(\mathrm{mg} / \mathrm{g})$} & $4.9 \pm 2.0$ & $22.7 \pm 5.6$ & $151.5 \pm 100.7$ & $1915 \pm 1101$ & $<0.0001$ \\
\hline \multicolumn{2}{|c|}{$\operatorname{GFR}\left(\mathrm{ml} / \mathrm{min} / 1.73 \mathrm{~m}^{2}\right)$} & $129.7 \pm 18.1$ & $113.1 \pm 11.5$ & $72.6 \pm 19.5$ & $23.1 \pm 14.8$ & $<0.0001$ \\
\hline \multicolumn{2}{|c|}{ NLR } & $1.9 \pm 0.30$ & $3.0 \pm 2.5$ & $2.2 \pm 1.5$ & $5.2 \pm 3.9$ & 0.007 \\
\hline \multicolumn{2}{|c|}{ PAI1 (ng/ml) } & $6.8 \pm 2.4$ & $7.7 \pm 1.8$ & $7.6 \pm 1.9$ & $8.7 \pm 1.2$ & 0.031 \\
\hline \multicolumn{2}{|c|}{ TIMP1 (ng/ml) } & $76.8 \pm 15.7$ & $91.4 \pm 19.8$ & $104.5 \pm 20.9$ & $120.6 \pm 18.2$ & 0.011 \\
\hline \multicolumn{2}{|l|}{ TPN } & $1.1 \pm 0.4$ & $1.2 \pm 0.3$ & $1.3 \pm 0.2$ & $1.8 \pm 0.3$ & $<0.0001$ \\
\hline
\end{tabular}

Abbreviations: A1: normo-albuminuria; A2: micro-albuminuria; A3: macro-albuminuria; HDL : high density lipoprotein; LDL : low density lipoprotein; FBS: fasting blood sugar; HBA1C ; hemoglobin A1 concentration; GFR: Glomerular filtration rate; NLR: neutrophil lymphocyte ratio; PAI-1 Plasminogen activator inhibitor 1; TIMP: Tissue inhibitor metalloproteinase-1,

$* \mathrm{p}<0.05$ is considered significant using Student's $\mathrm{t}$ test for comparison between A2 vs A3 
Table 2. Diagnostic performances of single and combined markers

\begin{tabular}{|c|c|c|c|c|c|c|c|}
\hline Variables & AUC & cutoff & Sensitivity & Specificity & PPV & NPV & Accuracy \\
\hline \multicolumn{8}{|c|}{ A. Normoalbuminuria group (A1) vs Microalbuminuria group (A2) } \\
\hline NLR & 0.41 & 1.9 & 42.9 & 46.7 & 27.3 & 63.6 & 45.5 \\
\hline TIMP-1 (ng/ml) & 0.72 & 96 & 71.4 & 66.7 & 50.0 & 83.3 & 68.2 \\
\hline PAI-1 (ng/ml) & 0.51 & 8.6 & 57.1 & 53.3 & 36.4 & 72.7 & 54.6 \\
\hline TPN & 0.61 & 1.34 & 64.3 & 63.3 & 45.0 & 79.2 & 63.6 \\
\hline \multicolumn{8}{|c|}{ B. Normoalbuminuria group (A1) vs Macroalbuminuria group (A3). } \\
\hline NLR & 0.72 & 2.9 & 67.7 & 70.0 & 71.9 & 65.6 & 68.8 \\
\hline TIMP-1 (ng/ml) & 0.88 & 109 & 81.8 & 83.3 & 84.4 & 80.7 & 82.5 \\
\hline PAI-1 (ng/ml) & 0.72 & 8.8 & 67.7 & 63.3 & 74.2 & 66.7 & 70.3 \\
\hline TPN & 0.88 & 1.48 & 81.8 & 80.0 & 81.8 & 80.0 & 81.0 \\
\hline \multicolumn{8}{|c|}{ C. Diabetic without nephropathy (A1) vs Diabetic with nephropathy (A2\&A3) } \\
\hline NLR & 0.63 & 2.5 & 60.4 & 56.7 & 69.1 & 47.2 & 59.0 \\
\hline TIMP-1 (ng/ml) & 0.83 & 101 & 76.6 & 73.3 & 81.8 & 66.7 & 75.3 \\
\hline PAI-1 (ng/ml) & 0.66 & 8.6 & 66.7 & 53.3 & 69.6 & 50.0 & 61.5 \\
\hline TPN & 0.80 & 1.42 & 70.2 & 70.0 & 78.6 & 60.0 & 70.1 \\
\hline \multicolumn{8}{|c|}{ D. Early stage of DN (A2) vs late stage of DN (A3). } \\
\hline NLR & 0.81 & 2.3 & 79.4 & 78.6 & 90.0 & 61.1 & 79.2 \\
\hline TIMP-1 (ng/ml) & 0.82 & 109 & 81.8 & 78.6 & 90.0 & 64.7 & 80.9 \\
\hline PAI-1 (ng/ml) & 0.74 & 8.8 & 67.7 & 64.3 & 82.1 & 45.0 & 66.7 \\
\hline TPN & 0.88 & 1.48 & 81.8 & 85.7 & 93.1 & 66.7 & 83.0 \\
\hline
\end{tabular}

Abbreviations: A1: normo-albuminuria; A2: micro-albuminuria; A3: macro-albuminuria; NLR: neutrophil lymphocyte ratio; PAI-1 Plasminogen activator inhibitor 1; TIMP: Tissue inhibitor metalloproteinase-1. 

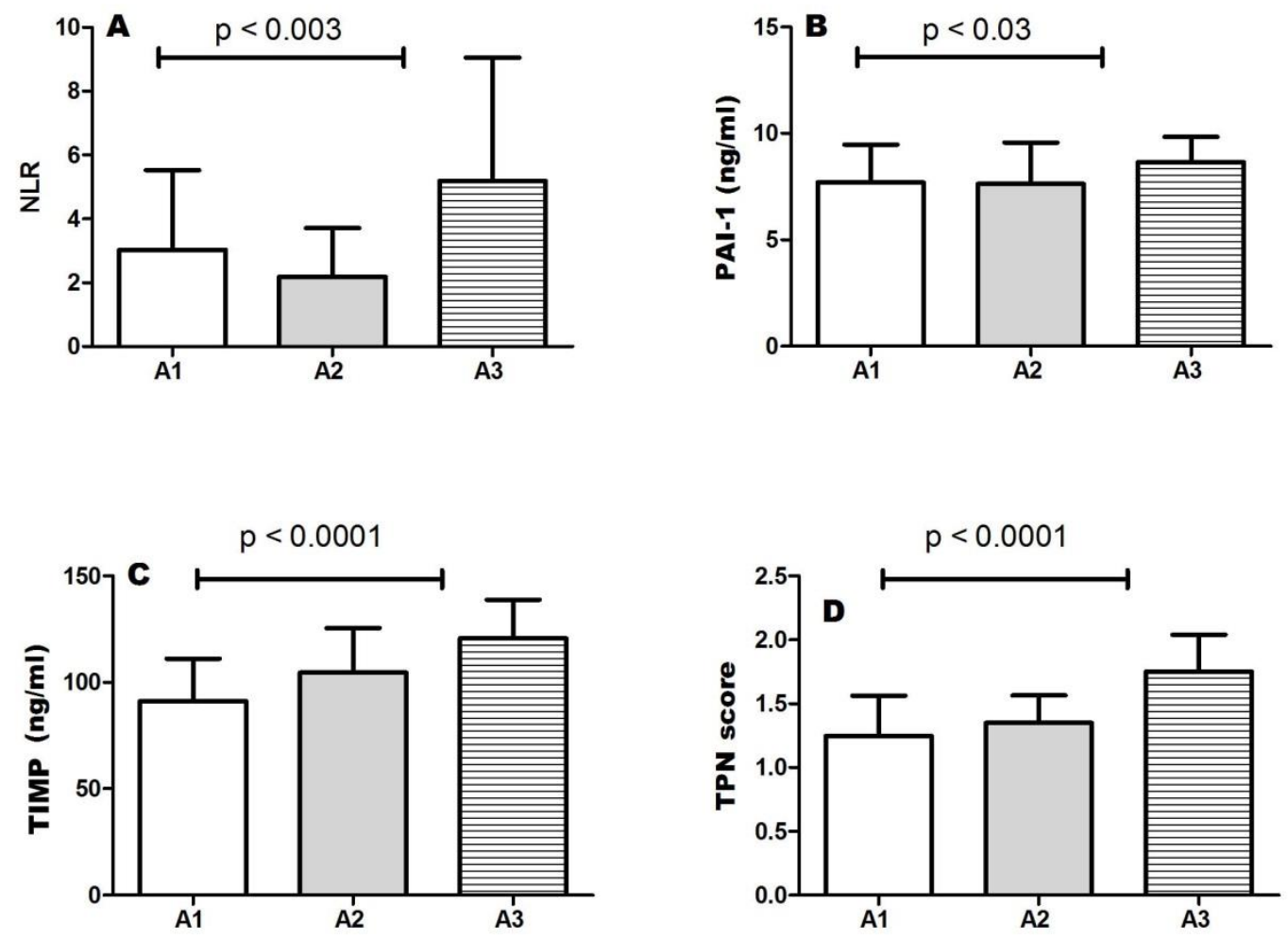

Figure 1. Levels of single candidate markers and combined markers in studied groups. A. level of neutrophil lymphocytes ratio. B. level of PAI1. C. level of TIMP1. D. level of combined three markers. $p<0.05$ is considered significant, and $\mathrm{p}<0.0001$ is considered extremely significant. 


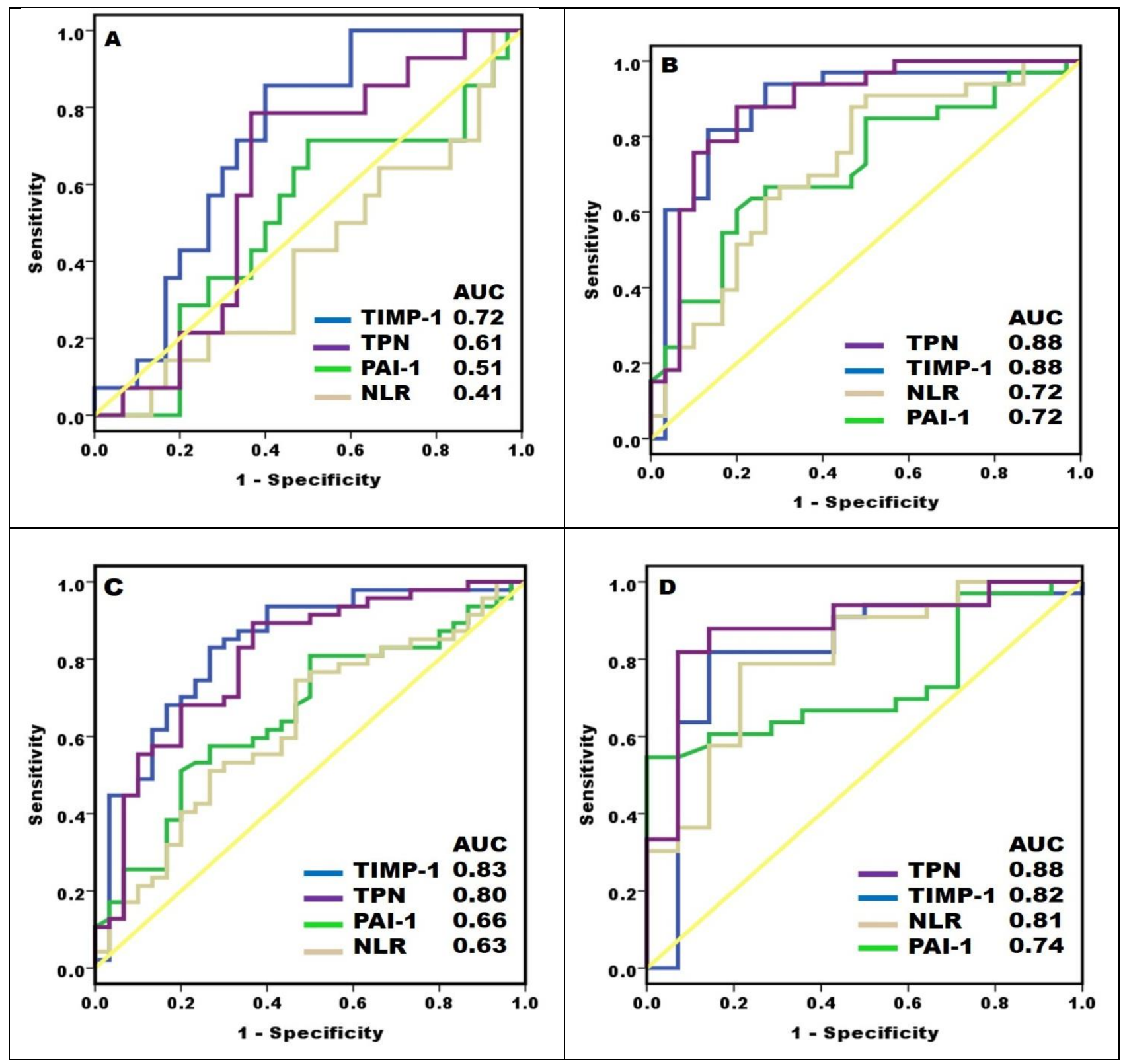

Figure 2. Diagnostic performance of candidate markers and combined three markers. A. Normoalbuminuria group (A1) vs microalbuminuria group (A2). B. Normoalbuminuria group (A1) vs macroalbuminuria group (A3). C. Diabetic without nephropathy (A1) vs diabetic with nephropathy (A2\&A3) D. Early stage of DN (A2) vs late stage of DN (A3). 


\section{Discussion:}

This present study examined the role of NLR, TIMP-1, and PAI-1 as biomarkers for the early detection and for evaluating the progression of DN. In this study, NLR values increased significantly with the severity of $\mathrm{DN}(\mathrm{p}=0.007)$. This finding agrees with several authors (Huang et al., 2017). DN is characterized by the progressive accumulation of ECM and ECM turnover regulation depends on the balance between its synthesis and degradation. The degradation of ECM involves the interaction of metalloproteinases with specific inhibitors such as TIMPs (Amanzadeh et al., 2018). ECM degradation and the activities of MMPs and TIMPs are affected by high glucose levels (Rysz et al., 2007). TIMPs and PAI-1 may be important in the development of diabetic nephropathy (McLennan et al., 2000; McLennan et al., 2004). In our study, the high levels of TIMP-1 were associated with diabetic nephropathy. TIMP-1 levels were increased in DM2 patients comparing to controls and TIMP-1 levels were significantly increased with advanced nephropathy (Lee et al., 2005; MoraGutierrez et al., 2018). In contrast, our study results don't agree with the results of Rysz et al (2007) which showed that TIMP-1 was significantly decreased in patients with diabetic nephropathy when compared with diabetic patients without nephropathy (Rysz et al., 2007). Plasminogen activator inhibitor-1 is an important multifunctional glycoprotein with impressive fibrosis promoting effects in chronic kidney diseases. Levels of PAI-1 were increased acute phase and chronic kidney diseases (Eddy, 2006). In the present study, PAI-1 levels were higher with a significant difference between chronic kidney diseases and diabetes. Our results were agreed with several studies (Xu et al., 2016). In contrast, another study showed that serum PAI-1 levels were lower in type 2 diabetic patients with macroalbuminuria comparing with those normoalbuminuria and microalbuminuria (Amanzadeh et al., 2018). In our study, using the
ROC curve, we found that TIMP-1 has the highest AUC, sensitivity, and specificity for DN comparing to PAI-1 and NLR to discriminate between diabetic patients with DN from diabetic patients; Our finding agrees with Al-Rubeaan et al. study which reported that PAI-1 had no any diagnostic value in DN patients (AUC< 0.6) (Al-Rubeaan et al., 2017). In the present study, NLR had AUC 0.81 with $79.4 \%$ sensitivity, 78.6 $\%$ specificity, and $79.2 \%$ accuracy to discriminate between A3 from A2. The AUC of NLR was 0.89 with $86 \%$ sensitivity and $69 \%$, specificity at cutoff 2 for predicting albuminuria in type 2DM (Chittawar et al., 2017). The AUC of NLR was 0.82 with $75 \%$ sensitivity and $93 \%$, specificity at cutoff 1.8 for predicting DKD in type 2DM (Huang et al., 2017). In the present study, The AUC of the TPN index was $0.61,0.88$, and 0.88 for discriminant diabetics with $\mathrm{A} 1$ from A2, A1 vs A3, and A2 vs A3. Li et al (2016) developed an index for end-stage renal disease in patients with type 2 diabetes based on vascular adhesion protein-1, $\mathrm{HbA} 1 \mathrm{c}$, estimated GFR, and proteinuria with AUC 0.94, 73\% sensitivity, and $94 \%$. In conclusion: TIMP- 1 is the most sensitive marker for discriminant DN stages than NLR and PAI-1. But the TPN index was the most efficient marker for discriminant diabetics patients with macro-albuminuria from patients with micro-albuminuria

\section{References:}

Al-Rubeaan, K., Siddiqui, K., Al-Ghonaim, M.A., Youssef, A.M., Al-Sharqawi, A.H., Al Naqeb. D. (2017). Assessment of the diagnostic value of different biomarkers in relation to various stages of diabetic nephropathy in type 2 diabetic patients. Sci Rep 7(1):2684.

Amanzadeh, M., Mota, A., Zarghami, N., AbediAzar, S., Abroon, S., Akbarian, N., Mihanfar, A., Rahmati-Yamchi, M. (2018). Association between matrix metalloproteinase-3 activity and glomerular 
filtration rate and albuminuria status in patients with type 2 diabetes mellitus. Iran J Kidney Dis 12(1):40-47. American Diabetes Association.

(2018). Classification and diagnosis of diabetes:

Standards of medical care in diabetes-2018.

Diabetes Care 41(1): S13-S27.

Baker, N.L., Hunt, K.J., Stevens, D.R., Jarai, G., Rosen, G.D., Klein, R.L., Virella, G., Lopes-Virella, M.F., DCCT/EDIC Research Group. (2018). Association between inflammatory markers and progression to kidney dysfunction: examining different assessment windows in patients with type 1 diabetes. Diabetes Care 41(1):128-135.

Chittawar, S., Dutta, D., Qureshi, Z., Surana, V., Khandare, S., Dubey TN. (2017). Neutrophillymphocyte ratio is a novel reliable predictor of nephropathy, retinopathy, and coronary artery disease in Indians with type-2 diabetes. Indian J Endocrinol Metab 21(6):864-870.

Dias, J.P., Shardell, M., Golden, S. H., Ahima, R.S., Crews, D.C. (2018). Racial/ethnic trends in prevalence of diabetic kidney disease in the United States. Kidney Int Rep 4(2): 334-337.

Eddy, A.A., Fogo, A.B. (2006). Plasminogen activator inhibitor-1 in chronic kidney disease: evidence and mechanisms of action. J Am Soc Nephrol 17(11):29993012 .

Fiseha, T. ( 2015). Urinary biomarkers for early diabetic nephropathy in type 2 diabetic patients. Biomark Res 3:16.

Guy, M., Borzomato, J.K., Newall, R.G., Kalra, P.A., Price, C.P. (2009). Protein and albumin-tocreatinine ratios in random urines accurately predict 24 $\mathrm{h}$ protein and albumin loss in patients with kidney disease. Ann Clin Biochem 46 (6):468-476.

Huang, L., Xie, Y., Dai, S., Zheng, H. (2017). Neutrophil-to-lymphocyte ratio in diabetic microangiopathy. Int J Clin Exp Pathol 10 (2):12231232.
Kim, Y., Park, C.W.

(2017).

New therapeutic agents in diabetic nephropathy. Korean J Intern Med 32(1):11-25.

Kuo, Y.T., Wang, Y.Y., Lin, S.Y., Chang, W.D. (2018). Age and sex differences in the relationship between neutrophil-to-lymphocyte ratio and chronic kidney disease among an adult population in Taiwan. Clin Chim Acta 486:98-103.

Lee SW, Song KE, Shin DS, Ahn SM, Ha ES, Kim DJ, Nam MS, Lee KW. (2005). Alterations in peripheral blood levels of TIMP-1, MMP-2, and MMP9 in patients with type- 2 diabetes. Diabetes Res Clin Pract 69 (2):175-179.

Li HY, Lin HA, Nien FJ, Wu VC, Jiang YD, Chang TJ, Kao HL, Lin MS,4, Wei JN, Lin CH, Shih SR, Hung CS, Chuang LM. (2016). Serum Vascular Adhesion Protein-1 Predicts End-Stage Renal Disease in Patients with Type 2 Diabetes. PLoS One 11(2):e0147981.

Levey, A.S., Inker, L.A., Coresh, J. (2014). GFR estimation: from physiology to public health. Am $\mathbf{J}$ Kidney Dis 63(5):820-834.

Małgorzewicz, S., Skrzypczak-Jankun, E., Jankun, J. (2013). Plasminogen activator inhibitor-1 in kidney pathology. Int J Mol Med 31(3):503-510.

McLennan, S.V., Fisher, E., Martell, S.Y., Death, A.K., Williams, P.F., Lyons, J.G., Yue, D.K.(2000). Effects of glucose on matrix metalloproteinase and plasmin activities in mesangial cells: possible role in diabetic nephropathy. Kidney Int Suppl 77: S81-S87.

McLennan, S.V., Wang, X.Y., Moreno. V., Yue. D.K., Twigg, S.M. (2004). Connective tissue growth factor mediates high glucose effects on matrix degradation through tissue inhibitor of matrix metalloproteinase type 1: implications for diabetic nephropathy. Endocrinol 145(12):5646-5655.

Mora-Gutierrez J M, Fernandez-Seara M A., Roblero M F S, Gonzalez O, Escalada F. J, Soler M 
J, Paramo J A and Garcia-Fernandez N. (2018). Matrix metalloproteinase-10 and tissue inhibitor of metalloproteinase-1 (TIMP-1) as early predictors of nephropathy in patients with type 2 diabetes mellitus. Nephrology Dialysis Transplantation 33 (1): i500.

Musial, K., Zwolińska, D. (2014). Pleiotropic functions of TIMP-1 in patients with chronic kidney disease. Cell Mol Life Sci 1(8):1547-1548.

Nah, E.H., Cho, S., Kim, S., Cho, H.I. (2017). Comparison of Urine Albumin-to-Creatinine Ratio (ACR) Between ACR Strip Test and Quantitative Test in Prediabetes and Diabetes. Ann Lab Med (1):28-33.

Polina, E.R., Araújo, R.R., Sbruzzi, R.C., Biolo. A., Rohde. L.E, Clausell. N., Dos Santos, K.G. (2018). Relationship of polymorphisms in the tissue inhibitor of metalloproteinase (TIMP)-1 and -2 genes with chronic heart failure. Sci Rep 8(1):9446.

Rysz, J., Banach, M., Stolarek, R.A, Pasnik, J., Cialkowska-Rysz, A., Koktysz, R., Piechota, M, Baj Z. (2007). Serum matrix metalloproteinases MMP-2 and MMP-9 and metalloproteinase tissue inhibitors TIMP-1 and TIMP-2 in diabetic nephropathy. J Nephrol 20(4):444-452.

Tatar, E., Mirili, C., Isikyakar, T., Yaprak, M., Guvercin, G., Ozay, E., Asci, G. (2016). The association of neutrophil/lymphocyte ratio and platelet/lymphocyte ratio with clinical outcomes in geriatric patients with stage 3-5 chronic kidney disease. Acta Clin Belg 71(4):221-226.

Vijay, S., Hamide, A., Senthilkumar, G.P., Mehalingam. V. (2018). Utility of urinary biomarkers as a diagnostic tool for early diabetic nephropathy in patients with type 2 diabetes mellitus. Diabetes Metab Syndr 12 (5):649-652.

Xu, F., Liu, H., Sun, Y. (2016). Association of plasminogen activator inhibitor-1 gene polymorphism and type 2 diabetic nephropathy. Ren Fail 38(1):157162.

Zeng, X.F., Lu, D.X., Li, J.M., Tan, Y., Li, Z., Zhou, L., Xi, Z.Q., Zhang, S.M., Duan, W. (2017)
Performance of urinary neutrophil gelatinaseassociated lipocalin, clusterin, and cystatin $\mathrm{C}$ in predicting diabetic kidney

disease and diabetic microalbuminuria: a consecutive cohort study. BMC Nephrol 18(1):233. 